\title{
Towards improving the MITC9 shell element
}

\author{
Klaus-Jürgen Bathe *, Phill-Seung Lee, Jean-François Hiller \\ Department of Mechanical Engineering, Massachusetts Institute of Technology, 77 Massachusetts Avenue Room 3-356, \\ Cambridge, MA 02139, USA
}

\begin{abstract}
Our objective in this paper is to present some results regarding the predictive capabilities of the MITC9 shell element when the tying points in the element are changed. The MITC9 element is a general nine-node shell element based on the formulation approach of using mixed-interpolated tensorial components. Different tying points are very simple to implement and are not decreasing the computational efficiency of the element. Hence, the use of the "best" tying points is clearly of value.
\end{abstract}

(c) 2003 Elsevier Science Ltd. All rights reserved.

\section{Introduction}

Finite element methods for the analysis of shell structures have been developed for almost half a century [1] and still improvements in the finite element procedures are very desirable. Shell structures can show a very varied and complex behavior and finite element methods that are general and always effective are very difficult to attain. However, the use of mixed methods has greatly advanced the field [1-3] and specifically, the mixedinterpolated shell elements proposed in Refs. [4-7] are employed.

During the recent years also mathematical insights and theories have been established that greatly help in assessing the effectiveness of finite element procedures $[3,8-10]$. These assessments in particular focus on whether a given finite element scheme is "uniformly optimal" for any shell structural problem as the shell thickness decreases.

We have evaluated in earlier publications the MITC shell elements regarding their predictive capabilities [1113] and concluded that while these elements are clearly powerful, improvements are still desirable, and in particular for the MITC9 shell element [13].

\footnotetext{
${ }^{*}$ Corresponding author. Tel.: +1-617-253-6645; fax: +1-617253-2275.

E-mail address: kjb@mit.com (K.J. Bathe).
}

In the next section of the paper we briefly summarize the important requirements for general shell elements. We then briefly mention in Section 3 the essence of the MITC9 shell element formulation and present the various modifications to the tying points tested in this study. The results obtained using these modifications in the solution of two rather tough test problems are then presented in Section 4, and we give our conclusions in Section 5 .

\section{On the assessment of shell finite element discretization schemes}

Our objective in this section is to briefly focus on the question as to how shell finite element methods should be tested. More details on this question are given in Refs. $[3,8,13]$.

\subsection{The spectrum of shell problems}

The difficulty of developing an effective general finite element solution scheme for any shell problem is due to the fact that shell structures can show totally different behaviors depending on the geometry, boundary conditions, and applied loading. We consider in this paper only linear analysis conditions; in non-linear analysis of course also the material conditions, whether or not contact is established, etc. would need to be considered [3]. 
Shell problems have been for a long time classified as membrane-dominated, bending-dominated and mixed problems. This classification has recently been made particularly precise by considering the asymptotic behaviors of shells. In the following we briefly summarize some important concepts, see [3,8-10].

Consider the shell mathematical model governed by equations that in variational form result in the problem statement

Find $\mathbf{u}^{\varepsilon} \in V$ such that

$\varepsilon^{3} A_{\mathrm{b}}\left(\mathbf{u}^{\varepsilon}, \mathbf{v}\right)+\varepsilon A_{\mathrm{m}}\left(\mathbf{u}^{\varepsilon}, \mathbf{v}\right)=\varepsilon^{\rho} G(\mathbf{v}) \quad \forall \mathbf{v} \in V$,

where $\varepsilon$ is the shell thickness parameter $t / L$ ( $t$ is the thickness and $L$ is a global characteristic dimension of the shell structure), the bilinear form $A_{\mathrm{b}}$ represents the scaled bending energy, the bilinear form $A_{\mathrm{m}}$ represents the scaled membrane and shear energies, $\mathbf{u}^{\varepsilon}$ is the unknown solution (displacement field), $\mathbf{v}$ is the test function, $V$ is the appropriate Sobolev space, and $G$ denotes the scaled external loading. It can be proven that, when a well-defined scaling exists, we have $1 \leqslant \rho \leqslant 3$.

The following subspace characterizes the asymptotic behavior of a shell as $\varepsilon$ approaches zero

$V_{0}=\left\{\mathbf{v} \in V \mid A_{\mathrm{m}}(\mathbf{v}, \mathbf{v})=0\right\}$.

If the content of this subspace is only the zero displacement field $\left(V_{0}=\{0\}\right)$, we say that "pure bending is inhibited" (or, in short, we have an "inhibited shell"). On the other hand, when the shell admits non-zero purebending displacements, we say that "pure bending is non-inhibited" (we have a "non-inhibited shell").
The "pure bending is non-inhibited" situation frequently results in the bending-dominated state and the appropriate value to use for the load-scaling factor $\rho$ is 3. The general form of the limit problem is

Find $\mathbf{u}^{0} \in V_{0}$ such that

$A_{\mathrm{b}}\left(\mathbf{u}^{0}, \mathbf{v}\right)=G(\mathbf{v}) \quad \forall \mathbf{v} \in V_{0}$.

Of course, this limit problem holds only when the loading activates the pure bending displacements; otherwise, the shell behavior is as for the "inhibited" case but very unstable.

In the case of the "pure bending is inhibited" situation, we use the load-scaling factor $\rho=1$. Then, provided the problem is well posed, we obtain the limit problem of the membrane-dominated case in the space $V_{\text {m }}$, a space larger than $V$, consisting of all displacements of bounded membrane (and shear) energy only

Find $\mathbf{u}^{\mathrm{m}} \in V_{\mathrm{m}}$ such that

$A_{\mathrm{m}}\left(\mathbf{u}^{\mathrm{m}}, \mathbf{v}\right)=G(\mathbf{v}) \quad \forall \mathbf{v} \in V_{\mathrm{m}}$.

This problem is well posed provided

$|G(\mathbf{v})| \leqslant C \sqrt{A_{\mathrm{m}}(\mathbf{v}, \mathbf{v})} \quad \forall \mathbf{v} \in V$

with $C$ a constant. This condition ensures that the applied loading can be resisted by membrane stresses only. If this is not possible, the asymptotic state does not correspond to membrane energy only, and the shell problem is classified as a mixed problem.

Hence, we have on the one side of the spectrum wellposed membrane-dominated problems and on the other side of the spectrum well-posed bending-dominated
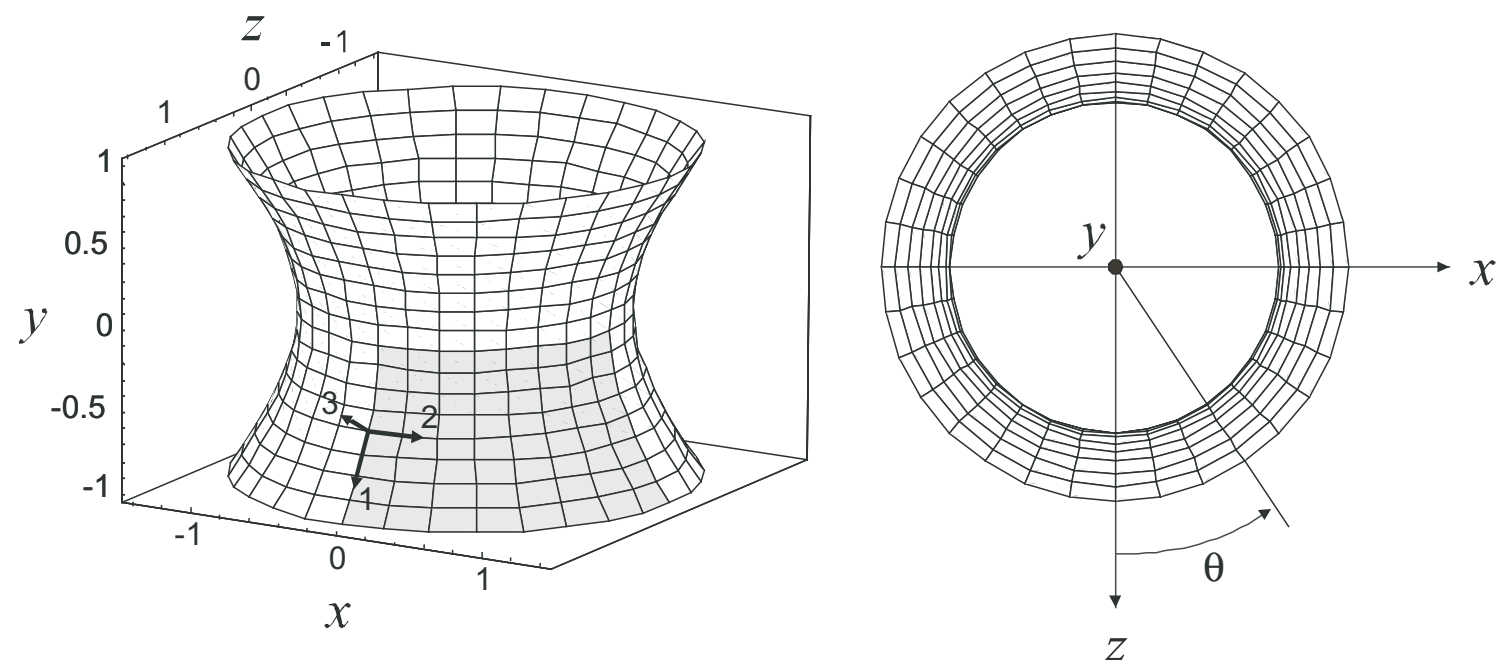

Fig. 1. The hyperboloid shell problem used with free ends and clamped ends (Young's modulus $=2.0 \mathrm{E} 11$, Poisson's ratio $=1 / 3$ ). The applied pressure is given in Eq. (10). 
problems. The difficulty in designing effective "general shell elements" is in obtaining elements that work equally well for both kinds of problems and, indeed, that are "uniformly optimal" for these problems. If this has been achieved, it is reasonable to assume that the finite element scheme will also work well for any other shell problem, including "monster-type problems" [10].

To have a "uniformly optimal shell element", we need the following convergence measure to hold $[1,3,13]$

$\frac{\left\|\mathbf{u}_{\mathrm{ref}}^{\varepsilon}-\mathbf{u}_{h}^{\varepsilon}\right\|_{*}}{\left\|\mathbf{u}_{\mathrm{ref}}^{\varepsilon}\right\|_{*}} \cong C h^{k}$

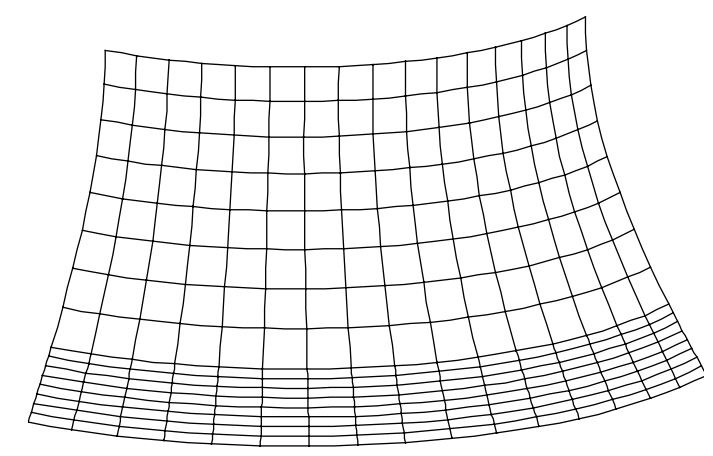

Fig. 2. Graded mesh $(16 \times 16, t=1 / 1000$, clamped case $)$.

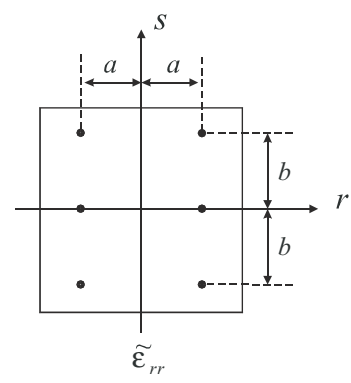

The strain interpolation functions are the 6-node element interpolation functions.

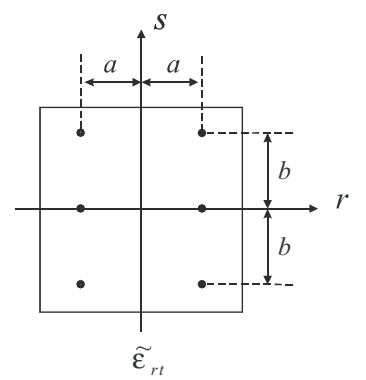

The strain interpolation functions are the 6-node element interpolation functions.

Fig. 3. Strain interpolations and tying points of the MITC9 shell element (the $\tilde{\varepsilon}_{s s}$ and $\tilde{\varepsilon}_{s t}$ components are interpolated in a symmetric manner) [1]. where $\mathbf{u}_{\mathrm{ref}}^{e}$ is the (accurate) reference solution usually replacing the exact solution $\mathbf{u}^{\varepsilon}, \mathbf{u}_{h}^{\varepsilon}$ is the finite element solution, $k$ is the displacement interpolation order, and $\|\cdot\|_{*}$ denotes the norm used. The constant $C$ must be independent of the shell thickness parameter $\varepsilon$ and the mesh sizes used, that is, the element size $h$.

To use in Eq. (6) the proper norm is crucial, since if the wrong norm is employed, the conclusions regarding
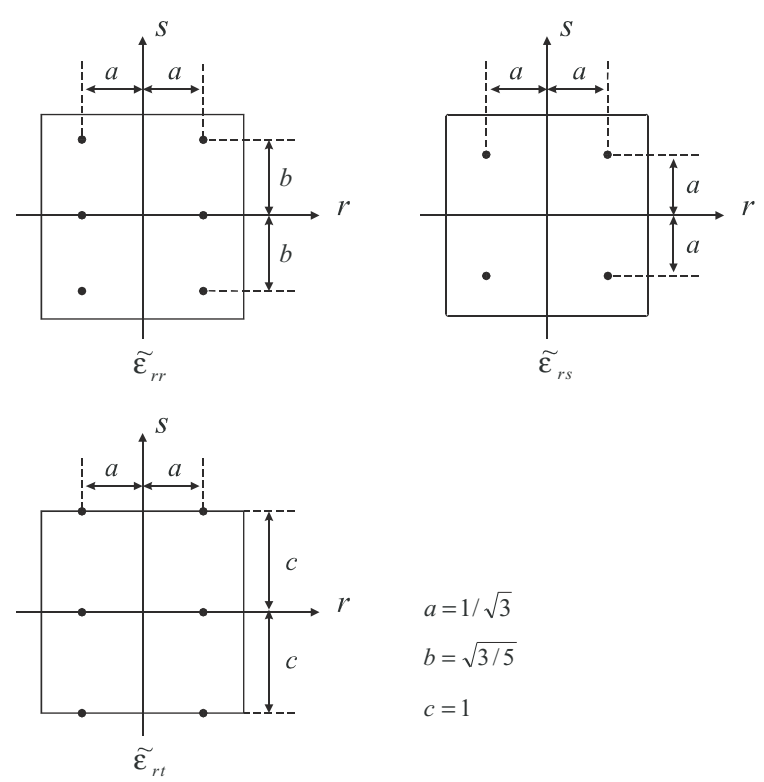

Fig. 4. Additional tying schemes of the MITC9 shell element.
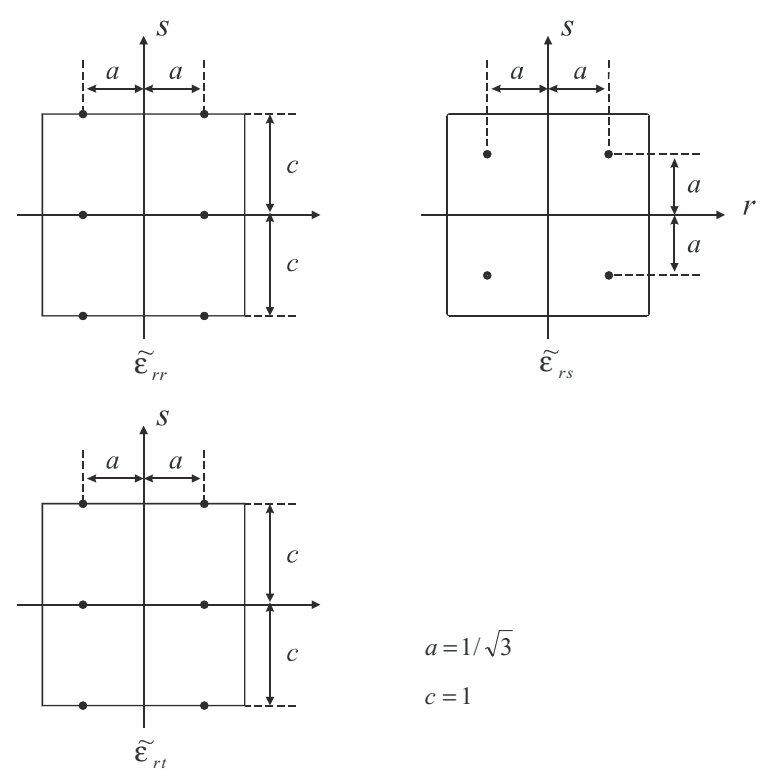

$$
\begin{aligned}
& a=1 / \sqrt{3} \\
& c=1
\end{aligned}
$$

Fig. 5. Additional tying schemes of the MITC9 shell element. 
the performance of the element scheme are certainly highly questionable and may well be wrong.

Since two different types of problems need to be considered, it is mathematically natural to use two different norms as discussed in Ref. [3]. However, we can also use the single norm proposed in Ref. [13], referred to below as the $s$-norm, and we shall use this norm in the following study. The $s$-norm is defined as
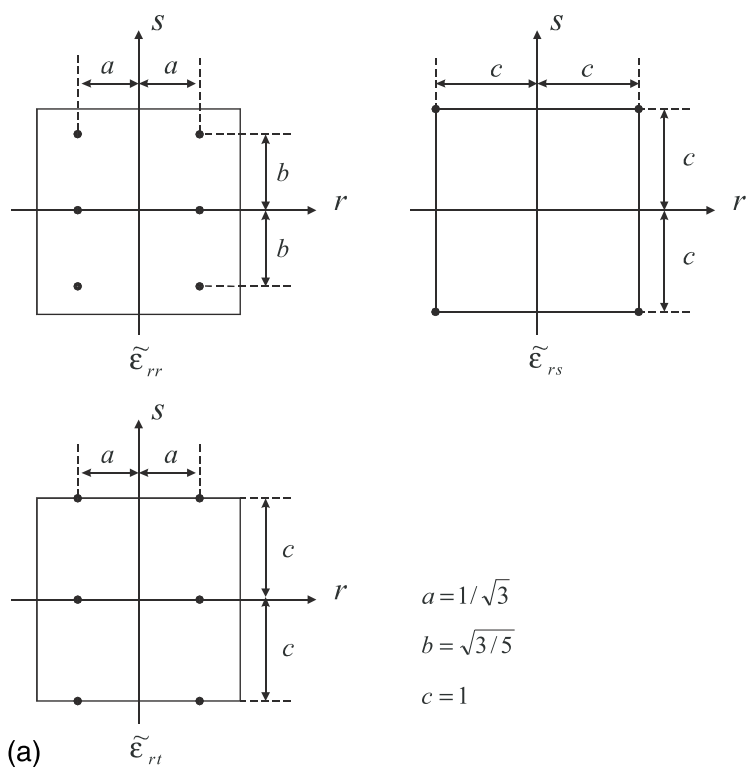

$$
\begin{aligned}
& a=1 / \sqrt{3} \\
& b=\sqrt{3 / 5} \\
& c=1
\end{aligned}
$$
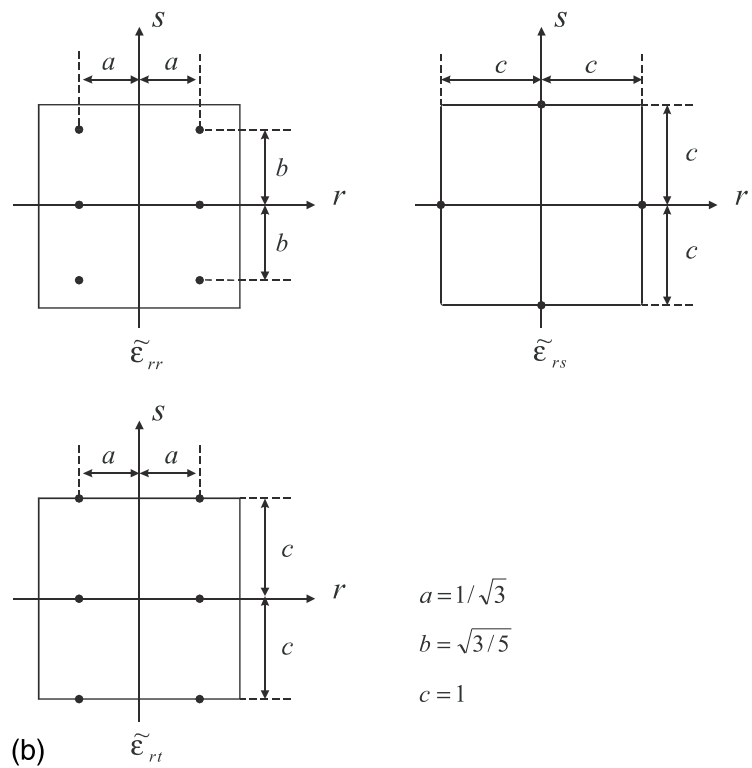

$$
\begin{aligned}
& a=1 / \sqrt{3} \\
& b=\sqrt{3 / 5} \\
& c=1
\end{aligned}
$$

Fig. 6. (a) Additional tying schemes of the MITC9 shell element (corner tying for $\tilde{\varepsilon}_{r s}$ ). (b) Additional tying schemes of the MITC9 shell element (edge tying for $\tilde{\varepsilon}_{r s}$ ).
$\left\|\mathbf{u}_{\mathrm{ref}}^{\varepsilon}-\mathbf{u}_{h}^{\varepsilon}\right\|_{s}^{2}=\int_{\Omega} \Delta \mathbf{e}^{\varepsilon \mathrm{T}} \mathbf{C}(\mathbf{x}) \Delta \mathbf{e}^{\varepsilon} \mathrm{d} \Omega$.

Here $\mathbf{C}(\mathbf{x})$ denotes the material law expressed in the Cartesian coordinate system (x), and denoting by $\mathbf{B}$ the strain operator of the continuum and by $\mathbf{B}_{h}$ the strain operator of the (mixed) finite element procedure

$$
\Delta \mathbf{e}^{\varepsilon}=\mathbf{B}(\mathbf{x}) \mathbf{u}_{\mathrm{ref}}^{\varepsilon}(\mathbf{x})-\mathbf{B}_{h}\left(\Pi^{-1}(\mathbf{x})\right) \mathbf{u}_{h}^{\varepsilon}\left(\Pi^{-1}(\mathbf{x})\right)
$$

with $\Pi$ defining a one-to-one mapping between every point of the discretization and the continuum. The advantage of using this norm lies in that it is physically based and can be employed for any shell problem. Also, let us note that if the finite element procedure is consistent, the strain operator $\mathbf{B}$ can be replaced by the finite element strain operator. For the discussion of this norm and results obtained with this norm we refer to Refs. $[3,13]$.
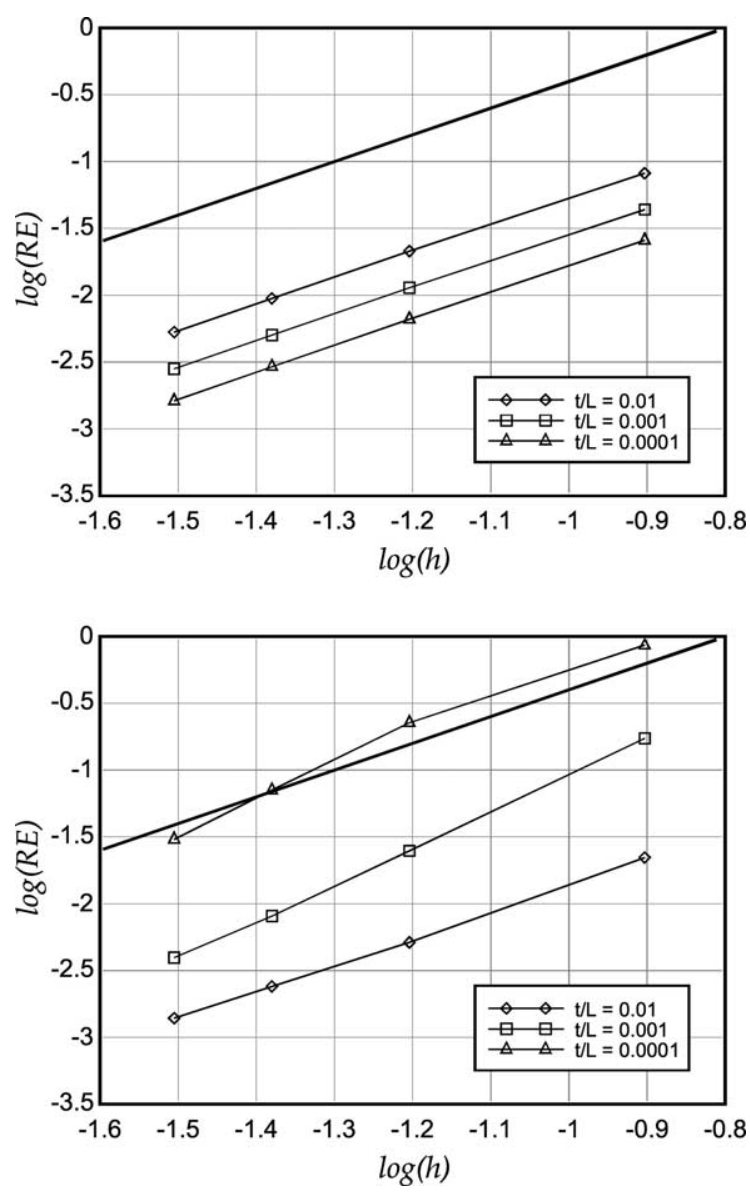

Fig. 7. Convergence curves of the MITC9 element using tying schemes in Fig. 3 (top: clamped case, bottom: free case). The bold line shows the optimal convergence rate, which equals 2.0. 
2.2. Considerations regarding element schemes and the test problems used

While shell analyses have been performed for decades and acceptable solutions in terms of some error assessment have been obtained, with advances in technology more stringent requirements have evolved and more accurate and efficient analyses of general shells are needed.

As summarized in the previous section, the analysis of general shells is difficult because of the different behaviors that can be encountered. It is extremely difficult to obtain a shell finite element method that is uniformly optimal for any shell problem and a mixed formulation

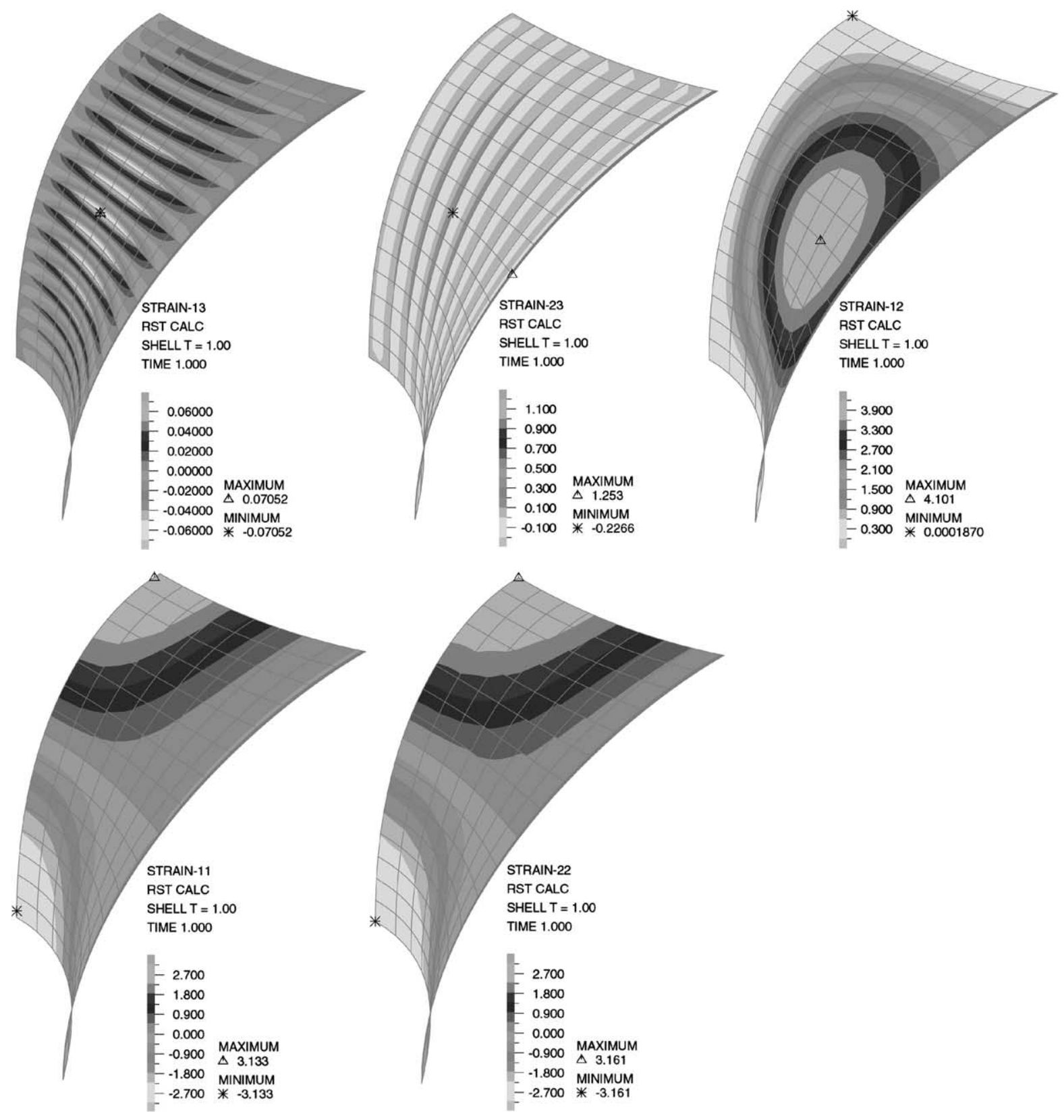

Fig. 8. Strain distributions on the deformed geometry of the free hyperboloid $(16 \times 16$ graded mesh, $t / L=1 / 1000)$; tying scheme of Fig. 3. The free edge is on the right. 
must be used. In this formulation we should aim to satisfy:

\section{Ellipticity}

Each shell finite element and the complete finite element discretization, when not supported, must always exactly contain only the six physical zero eigenvalues, corresponding to the rigid body modes, and not any less or additional zero eigenvalues. We cannot recommend the use of any element for general shell analyses that violates this condition, see Ref. [1, p. 473].

\section{Consistency}

The discretization scheme must be consistent in representing all strain terms. Briefly, this means that the bilinear forms used in the finite element discretization,

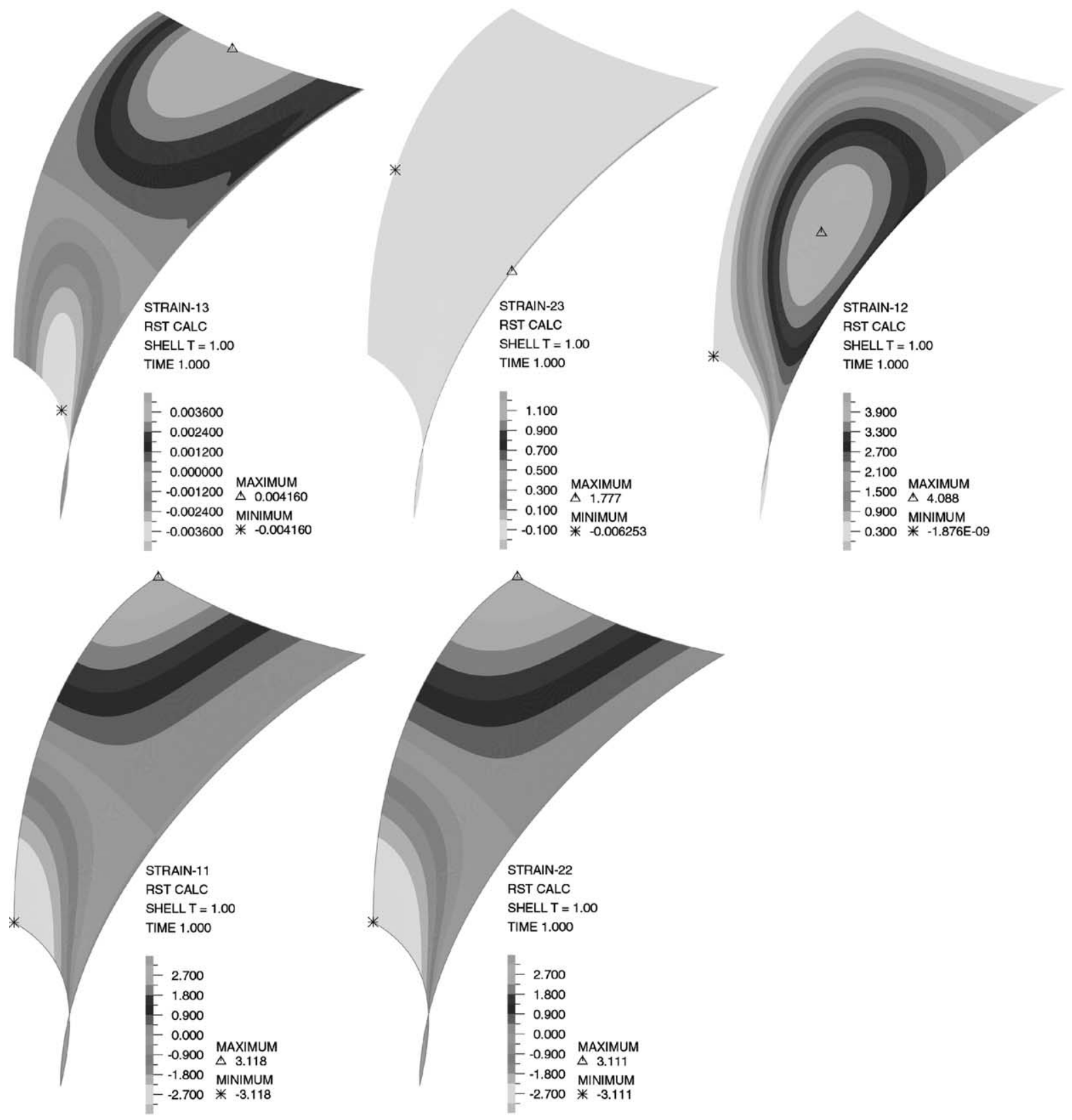

Fig. 9. Reference strain distributions of the free hyperboloid $(96 \times 96$ graded mesh, $t / L=1 / 1000)$. 
which may be a function of the element size $h$, must approach the exact bilinear forms of the mathematical model as $h$ goes to zero.

\section{Inf-sup condition}

Ideally, the mixed finite element scheme would satisfy the inf-sup condition, see Refs. $[1,3,14]$. Elements that satisfy this condition would be "uniformly optimal", as defined in Eq. (6), in bending-dominated problems for the interpolations used. However, no general shell finite element scheme has so far been proven analytically to satisfy the relevant inf-sup condition. So far only numerical tests have been performed on general shell discretization schemes [12,14].
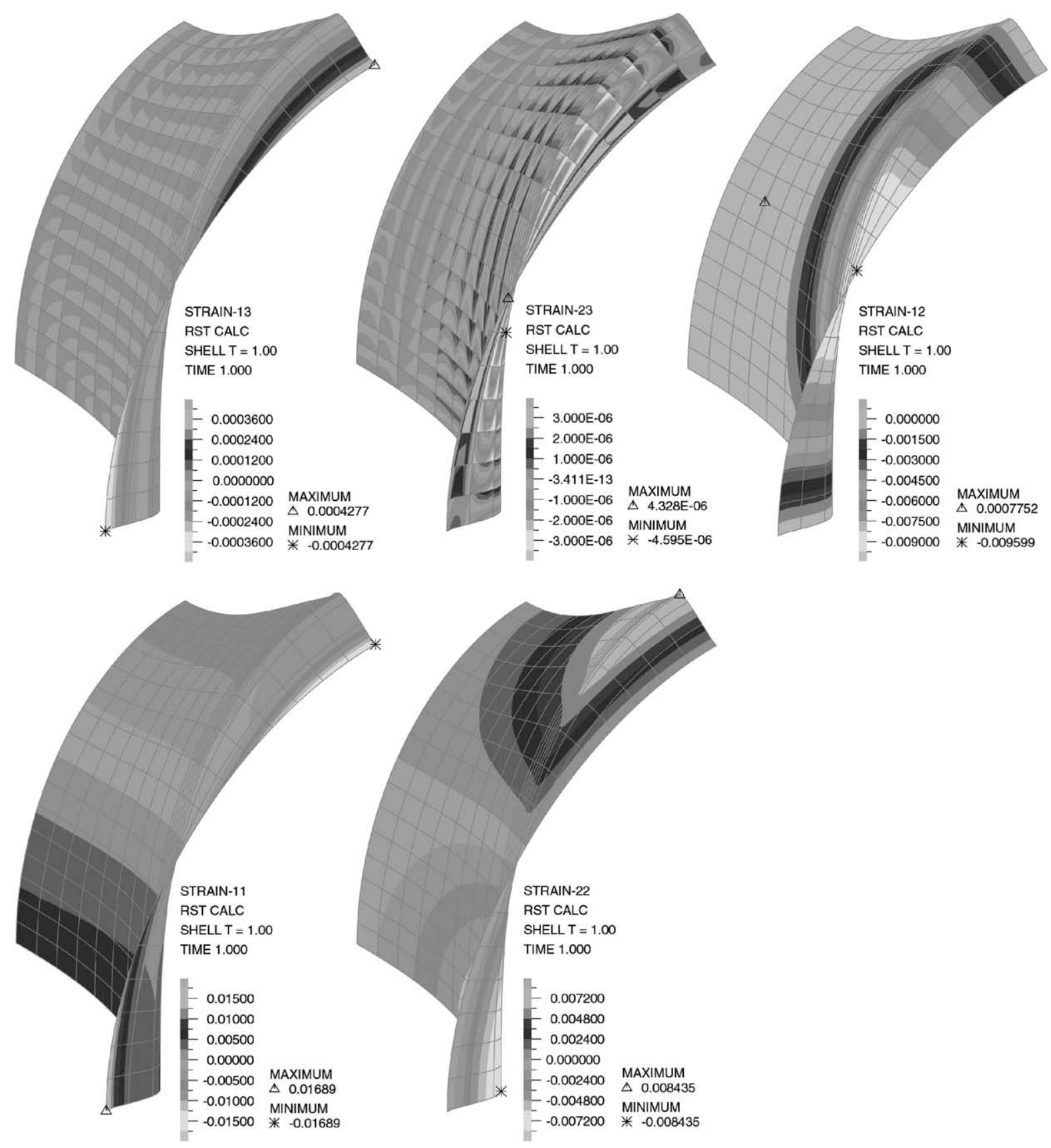

Fig. 10. Strain distributions on the deformed geometry of the clamped hyperboloid $(16 \times 16$ graded mesh, $t / L=1 / 1000)$; tying scheme of Fig. 3. The clamped edge is on the right. 
Since rigorous mathematical investigations on whether a general shell finite element scheme is uniformly optimal are still out of reach, recourse to numerical investigations is largely necessary. However, the numerical studies must be based upon the general mathematical understanding available and need to be well designed.

In Ref. [13] we used two shell problems in order to evaluate shell discretization schemes. The problems are depicted in Fig. 1. The shell is an axisymmetric hyperboloid structure subjected to a smoothly varying pressure. The surface is defined as

$x^{2}+z^{2}=1+y^{2} ; \quad y \in[-1,1]$

and the loading imposed is the periodic pressure

$p(\theta)=P_{0} \cos (2 \theta)$.
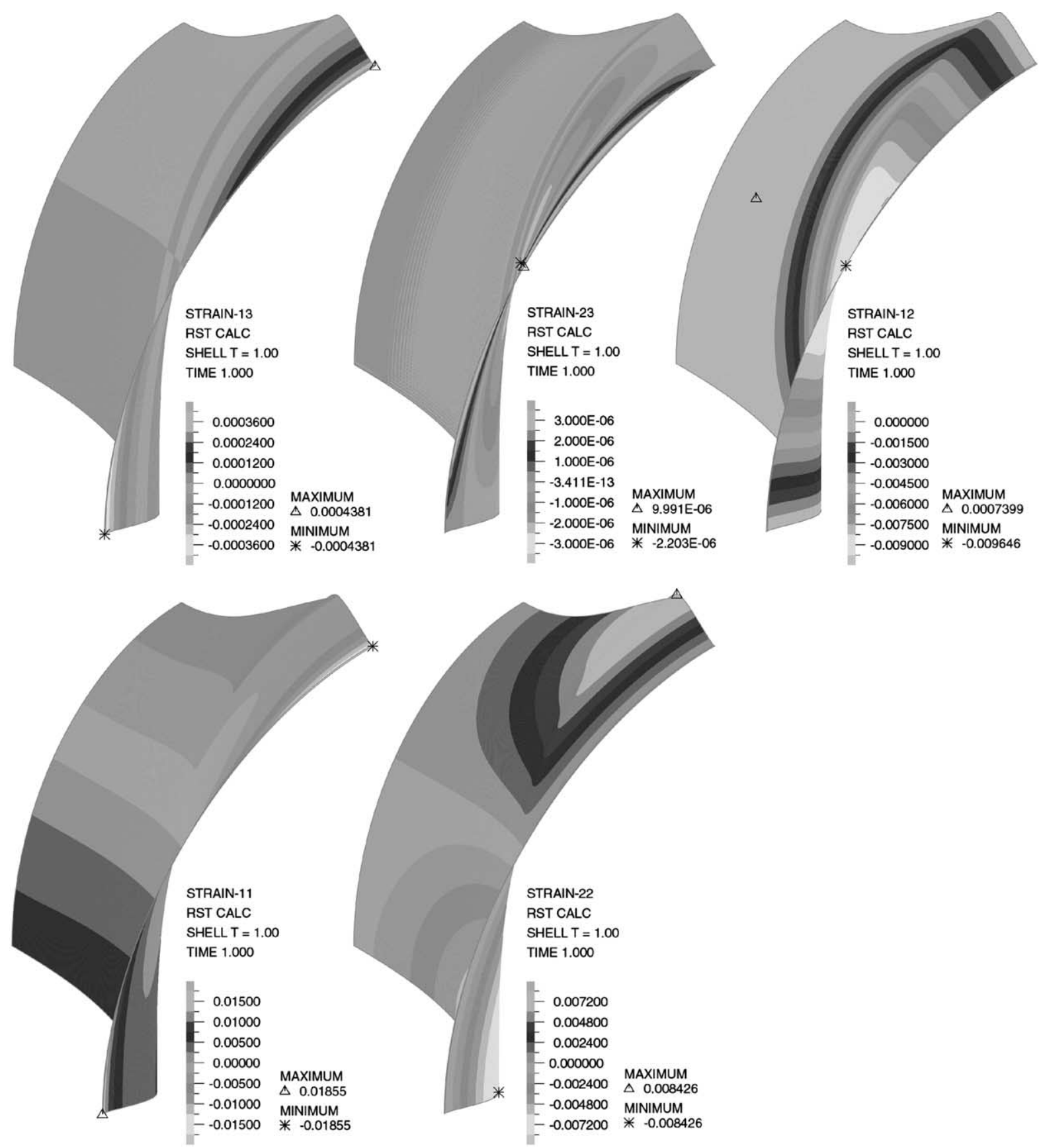

Fig. 11. Reference strain distributions of the clamped hyperboloid $(96 \times 96$ graded mesh, $t / L=1 / 1000)$. 
A bending-dominated problem (corresponding to $\rho=3$ ) is obtained when the ends are free and a membranedominated problem (corresponding to $\rho=1$ ) is obtained when the ends are clamped, see Ref. [13]. We shall use the same problems also in the study presented below.

An important requirement in the convergence studies is to mesh the boundary layers appropriately if we want to observe the actual and proper convergence rates of finite element discretizations. The meshing used should ideally be such that the error measured per unit volume or surface of the shell is uniform in the finite element solutions [1].

For the two problems considered here, we are using the same meshes as in Ref. [13] because these have been identified to be appropriate meshes. Hence, we use symmetry conditions and consider one-eighth of the structure (that is, half the length and $90^{\circ}$ ), and $L=1$ in Eq. (1). Since no analytical solutions are available we employ in each case a reference solution using a very fine mesh to measure the errors obtained using the coarser meshes. For the reference solutions a 96 by 96 element mesh of MITC9 shell elements is used, and the convergence and accuracy studies are performed using meshes of sizes $8 \times 8,16 \times 16,24 \times 24$, and $32 \times 32$.

In each case, half the mesh is used to represent the boundary layer as shown in Fig. 2. For the clamped hyperboloid, the boundary layer thickness used is $6 \sqrt{t}$, and for the free hyperboloid the boundary layer thickness used is $0.5 \sqrt{t}$ [13].

\section{The MITC9 element and tying points used}

The MITC9 element has been described in various publications $[1,3,7,11]$ and there is no need to describe the element here again. The key point is that in the MITC element formulations the covariant strain components are interpolated and then tied to the displacements. Hence, only the usual displacements and rotations at the nodes are used as degrees of freedom. For the MITC9 element, the usual nine nodes for Lagrangian displacement interpolations are employed.

Fig. 3 gives the strain interpolations and tying points used for the MITC9 shell element proposed in Ref. [7]. Of course, alternative tying points (for the same interpolations) are possible, and in particular those shown in Fig. 4. In addition, we can consider using the tying points shown in Figs. 5 and 6. However, the element based on the tying points given in Figs. 6(a) and (b), respectively, contains a spurious zero energy mode and shows locking, and so we do not consider these two alternatives any further.

Indeed, the MITC9 element using the tying points in Fig. 5 displays in essence the same predictive capability as when the tying points in Fig. 4 are used, with the tying points of Fig. 4 resulting in slightly better results. Hence the real comparison is between the use of the tying points shown in Figs. 3 and 4. For the tying points of Figs. 4 and 5 the MITC9 element does not contain any spurious zero energy mode (the ellipticity condition is satisfied) and the element is consistent in the sense discussed above [13].

In practice and in our studies below, the MITC9 shell element is used as an element degenerated from threedimensional continuum mechanics, and hence the underlying shell theory is not exactly of the form used in Eq. (1), but as the shell thickness decreases the problems considered in Section 2.1 are reached, see $[3,15]$.

\section{Numerical results}

As mentioned above we are solving the two problems of Fig. 1. These are a membrane-dominated problem and a bending-dominated problem. In all solution cases we use the graded meshes described above.

Fig. 7 shows the convergence results obtained for the MITC9 element with the tying in Fig. 3. RE in the figure is the relative error measure
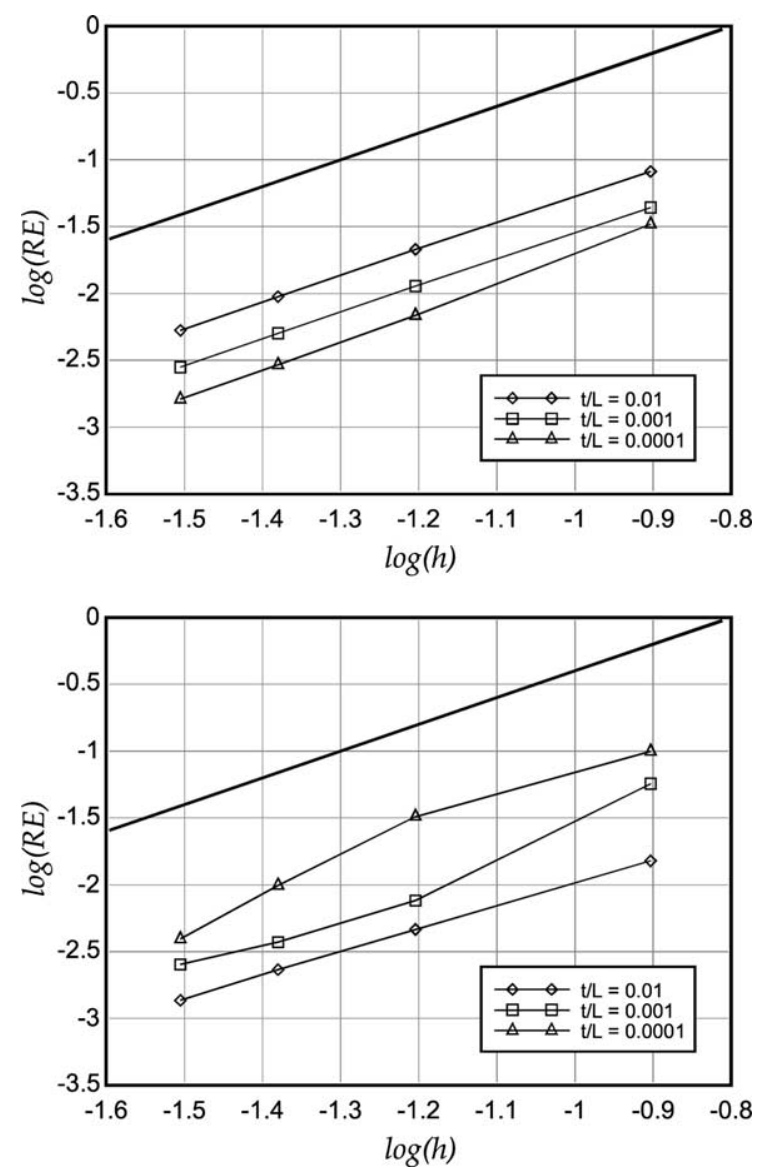

Fig. 12. Convergence curves of the MITC9 element using tying schemes in Fig. 4 (top: clamped case, bottom: free case). 


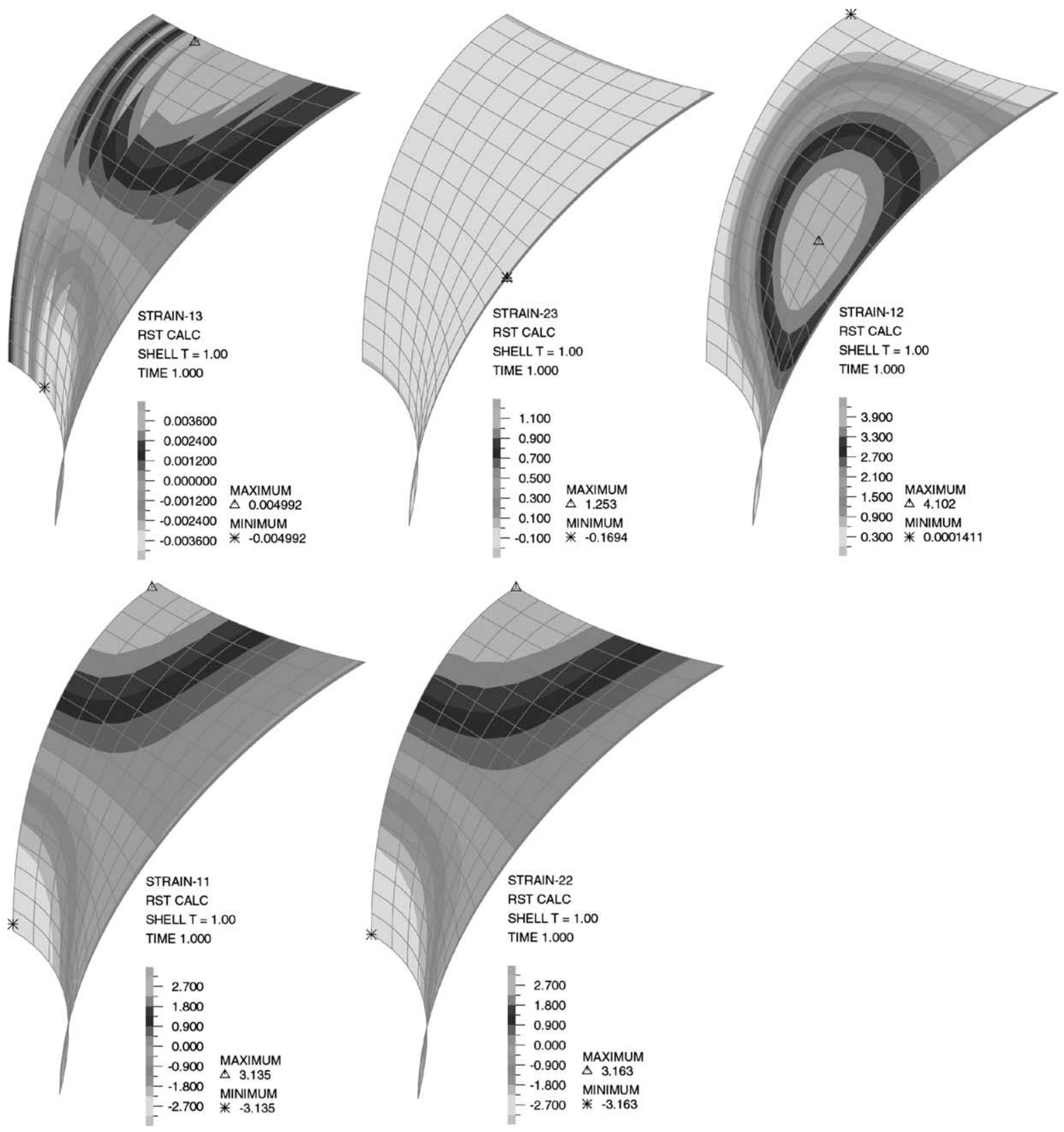

Fig. 13. Strain distributions of the free hyperboloid $(16 \times 16$ graded mesh, $t / L=1 / 1000)$; tying scheme of Fig. 4 .

$\mathrm{RE}=\frac{\left\|\mathbf{u}_{\mathrm{ref}}^{\varepsilon}-\mathbf{u}_{h}^{\varepsilon}\right\|_{s}}{\left\|\mathbf{u}_{\mathrm{ref}}^{\varepsilon}\right\|_{s}}$

These results were already reported in Ref. [13] and actually spurred this further study. The convergence for the clamped structure is "uniformly optimal" in the sense that the convergence curves show the optimal slope and do not shift upward as the shell thickness is decreased. Indeed, the curves shift downward which is better than what we require. However, the convergence curves for the free structure do not show a uniformly optimal behavior. The optimal slope is present but there is a marked shift of the curves upward as the shell thickness is decreased. Clearly we should aim to improve upon this behavior.

In order to identify why the shifting of the curves occurs we study the predicted strain values for the shell thickness $t=1 / 1000$. Figs. 8 and 9 show, respectively, 

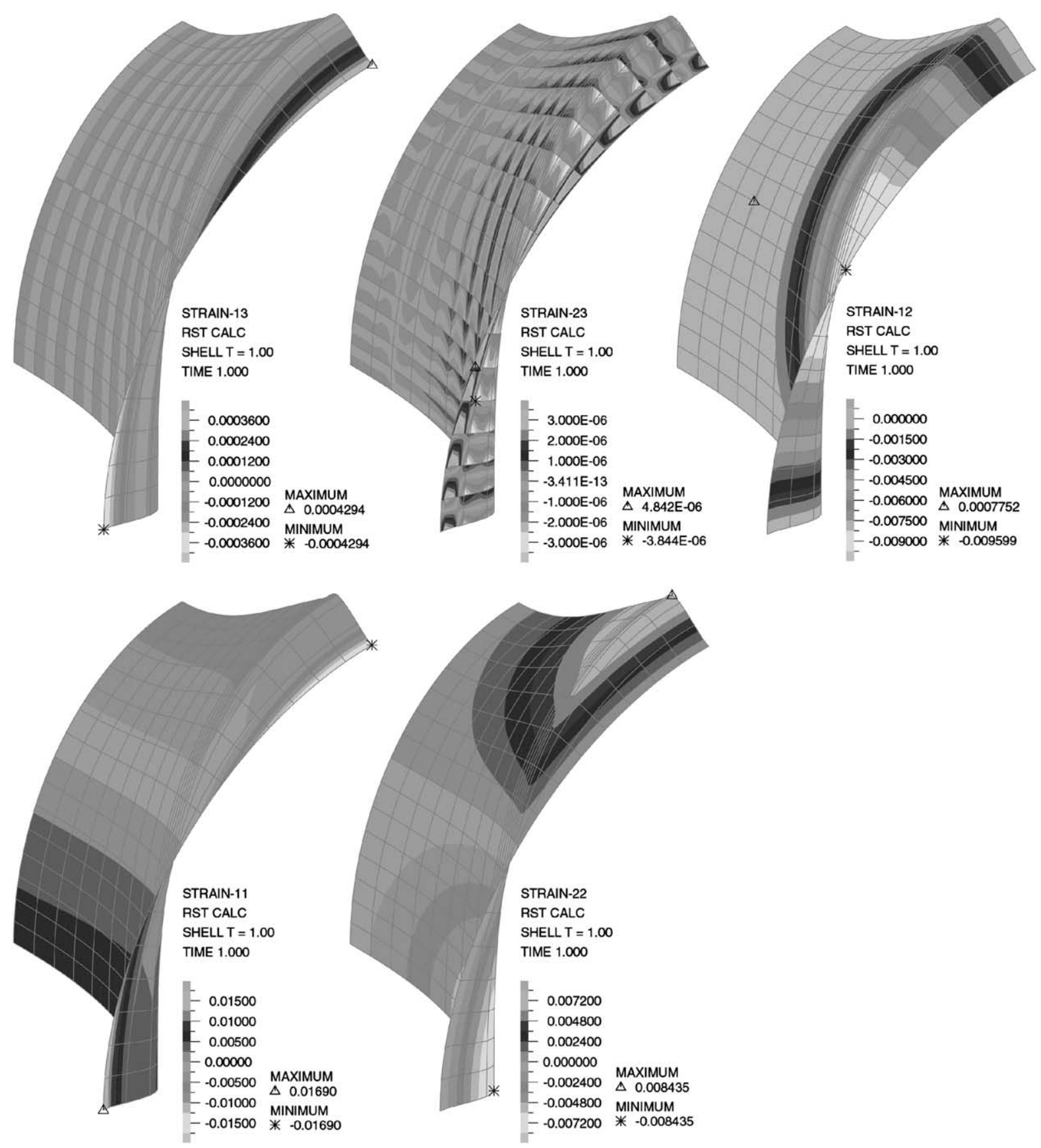

Fig. 14. Strain distributions of the clamped hyperboloid $(16 \times 16$ graded mesh, $t / L=1 / 1000)$; tying scheme of Fig. 4 .

the calculated strain values using the $16 \times 16$ element mesh and the reference values for the free edge case. All strain values given in the figures of this paper are unsmoothed [1] for the outer surface of the shell and correspond to the orthogonal axes in the plane of the shell shown in Fig. 1, see Ref. [1, p. 438]. We see that the strain components are well predicted using the $16 \times 16$ element mesh except for the transverse shear strain components.

For comparison we also show the strain values for the clamped edge case. The strain results calculated with the $16 \times 16$ element mesh are quite accurate, see Fig. 10, when compared to the reference values, see Fig. 11. 

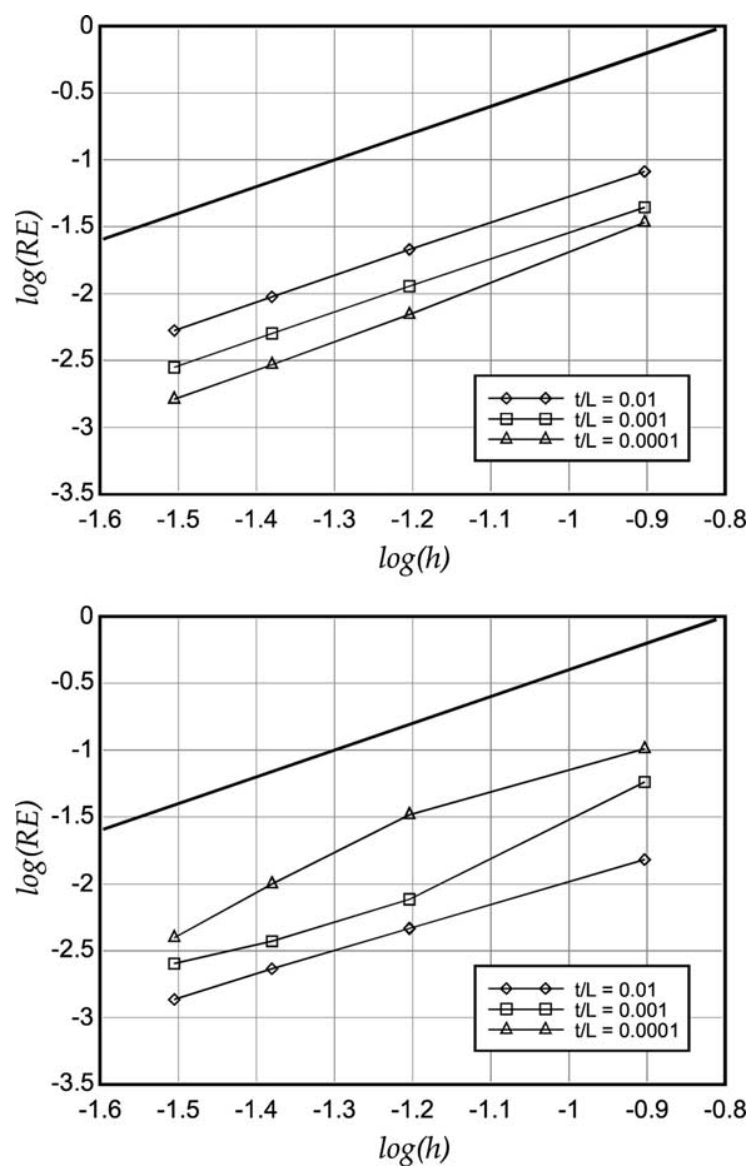

Fig. 15. Convergence curves of the MITC9 element using tying schemes in Fig. 5 (top: clamped case, bottom: free case).

Fig. 12 shows the convergence results obtained for the MITC9 element with the tying points in Fig. 4. The convergence curves for the clamped structure are essentially as in Fig. 7, but the convergence curves for the free structure show a significant improvement. The slope is still the optimal slope and significantly less shifting is present as the shell thickness decreases.

Fig. 13 shows the calculated strain values obtained with the same coarse $16 \times 16$ element mesh. Clearly, the results for the transverse shear strain components are now much better and the other strain component solutions have hardly changed. Fig. 14 shows also the strain values for the clamped case and we see that these are still quite accurate as well.

Finally, we show in Fig. 15 the convergence results obtained using the tying points of Fig. 5. We see that there is little difference to the results given in Fig. 12. The predicted strain values (not shown) are also quite close to those shown in Figs. 13 and 14.

Based on these observations, we conclude that for the axisymmetric hyperboloid problem with the edges free, the MITC9 element using the tying points in Fig. 4 has significantly better predictive capabilities than when using the tying points in Fig. 3.

\section{Concluding remarks}

Our objective in this paper was to present some results using the MITC9 shell element when the tying points for the strain components are changed.

The MITC9 shell element using the tying points earlier proposed [7] is clearly a powerful element and has shown excellent convergence behavior in many problem solutions. In the rather difficult doubly-curved shell problems considered in this paper, the element is also optimal, but only "uniformly" optimal when the shell is clamped. A study of the predicted strain components shows that the transverse shear strains are not predicted to sufficient accuracy when the shell is free at its edges.

A change of the tying points for the shear strain components to the element edges results into significantly better results. Optimality in the convergence behavior is retained and all strain components are predicted with reasonable accuracy. The convergence curves show much less shifting as the shell thickness is decreased.

Other options of tying points were also considered but did not result into a further improvement in the predictive capability of the element.

Of course, further studies of the element should be performed, both analytically as far as possible and also numerically using additional test problems (see Ref. [3] and the references therein). However, when performing numerical tests the strategy used in this paper is very valuable.

\section{References}

[1] Bathe KJ. Finite element procedures. New York: Prentice Hall; 1996.

[2] Bathe KJ, editor. Computational fluid and solid mechanics. Proceedings of the First MIT Conference on Computational Fluid and Solid Mechanics. Amsterdam: Elsevier Science; 2001.

[3] Chapelle D, Bathe KJ. The finite element analysis of shells-fundamentals. Berlin: Springer-Verlag, 2003.

[4] Dvorkin EN, Bathe KJ. A continuum mechanics based four-node shell element for general nonlinear analysis. Eng Comput 1984;1:77-88.

[5] Bathe KJ, Dvorkin EN. A 4-node plate bending element based on Mindlin/Reissner plate theory and a mixed interpolation. Int J Numer Meth Eng 1985;21:367-83.

[6] Bathe KJ, Dvorkin EN. A formulation of general shell elements - the use of mixed interpolation of tensorial components. Int J Numer Meth Eng 1986;22:697-722.

[7] Bucalem M, Bathe KJ. Higher-order MITC general shell elements. Int J Numer Meth Eng 1993;36:3729-54. 
[8] Chapelle D, Bathe KJ. Fundamental considerations for the finite element analysis of shell structures. Comput Struct 1998;66(19-36):711-2.

[9] Lee PS, Bathe KJ. On the asymptotic behavior of shell structures and the evaluation in finite element solutions. Comput Struct 2002;80:235-55.

[10] Bathe KJ, Chapelle D, Lee PS. A shell problem 'highly sensitive' to thickness changes. Int $\mathrm{J}$ Numer Meth Eng, in press.

[11] Bathe KJ, Iosilevich A, Chapelle D. An evaluation of the MITC shell elements. Comput Struct 2000;75:1-30.
[12] Bathe KJ, Iosilevich A, Chapelle D. An inf-sup test for shell finite elements. Comput Struct 2000;75:439-56.

[13] Hiller JF, Bathe KJ. Measuring convergence of mixed finite element discretizations: an application to shell structures. Comput Struct, in press.

[14] Bathe KJ. The inf-sup condition and its evaluation for mixed finite element methods. Comput Struct 2001;79:24352, 971.

[15] Chapelle D, Bathe KJ. The mathematical shell model underlying general shell elements. Int J Numer Meth Eng 2000;48:289-313. 